\title{
COMMUNICATION
}

\section{Artin and Levin Awarded National Medal of Science}

\author{
Elaine Kehoe
}

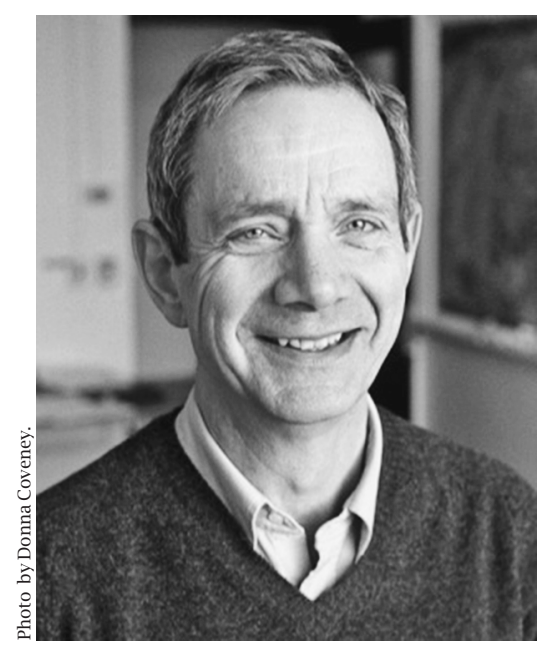

Michael Artin
Michael ARTin of the Massachusetts Institute of Technology and SIMON LEVIN of Princeton University have been honored with the 2015 National Medal of Science, the United States' highest honor for achievement in science.

The Work of Michael Artin

The Notices asked David Harbater of the University of Pennsylvania to comment on the work of Artin. Harbater responded: "Michael Artin is one of the founders of modern algebraic geometry, developing, together with Grothendieck, the notions of Grothendieck topology and étale cohomology, which were later key to the proofs of the Weil conjectures and many other developments. By introducing the notions of algebraic spaces and algebraic stacks, he created a context that permitted algebraic geometers to go beyond the limitations of schemes. Combined with his approximation and algebraization theorems, this led to representability results for algebraic spaces and to existence theorems for moduli spaces of algebraic and geometric objects. These developments provided foundations for a more modern deformation theory, including the important notion of versal deformation in the study of local moduli.

"His opus has also included major developments in more classical settings, such as his fundamental contributions to the study of surface singularities,

For permission to reprint this article, please contact:

reprint-permission@ams .org.

DOI: http://dx.doi.org/10.1090/noti1385 particularly the notion of rational singularity. He also found a striking example, with Mumford, of a unirational variety that is not rational, and in joint work with Swinnerton-Dyer proved the Shafarevich-Tate conjecture for elliptic curves over rational function fields whose minimal models are K3 surfaces. Other work of his related algebraic geometry to topology, including

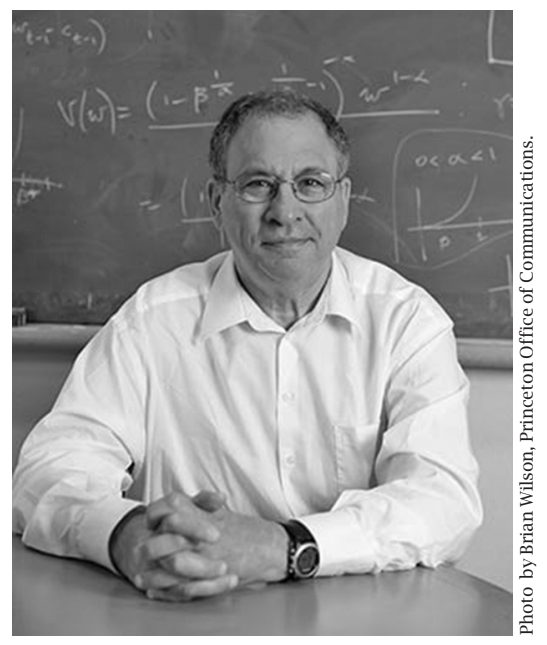

Simon Levin his work with Mazur on étale homotopy and the study of diffeomorphisms of compact manifolds.

"In a quite different direction, he brought ideas of algebraic geometry to bear on noncommutative algebra, dramatically changing the landscape in that area. This work included both the study of noncommutative algebras associated to schemes and also the introduction of noncommutative analogs of schemes that synthesize geometric notions with the theory of associative algebras. He has influenced generations of students, not only through his mentoring but also through his innovative and widely used textbook Algebra (1991), which was based on his classic course at MIT."

Michael Artin was born in Hamburg, Germany, the son of mathematician Emil Artin. He received his $\mathrm{PhD}$ from Harvard University in 1960 before joining MIT in 1963. He served as AMS president from 1991 to 1992 and was awarded the Society's Steele Prize for Lifetime Achievement in 2002, the Harvard Centennial Medal in 2005, and the Wolf Prize in 2013. Artin is a member of the National Academy of Sciences and a fellow of the AMS, the Society 
for Industrial and Applied Mathematics (SIAM), the American Academy of Sciences, and the American Association for the Advancement of Science.

\section{The Work of Simon Levin}

The Notices asked Alan Hastings, Distinguished Professor of Environmental Science and Policy at the University of California, Davis, to comment on the work of Levin.

Hastings responded: "Simon Levin worked on partial differential equations in his $\mathrm{PhD}$ thesis, but almost immediately turned his attention to the emerging field of mathematical biology. He has been a leader in many ways, including serving as the co-editor in chief of the Journal of Mathematical Biology. His research has used ideas from a variety of mathematical fields to look at problems in spatial ecology, complex adaptive systems, and, more recently, issues of ecological economics and financial systems. Levin has been a leader in using mathematical approaches to scale from microscopic descriptions of ecological systems to macroscopic descriptions, including spatial stochastic approaches. He has specifically studied problems ranging from plant communities to the movement of bird flocks to dynamics of infectious diseases to human decision making. His work has been characterized by the ability to develop models of just the right complexity-complex enough to describe the ecological phenomena yet simple enough to allow mathematical study."

Simon Levin received his $\mathrm{PhD}$ in mathematics from the University of Maryland in 1964. He was affiliated with Cornell University from 1965 to 1992, when he joined the faculty of Princeton University. He has received the Okubo Award of the Society for Mathematical Biology and the Japanese Society for Mathematical Biology (2001) and the Kyoto Prize in Basic Sciences in 2005. He is a fellow of the American Academy of Arts and Sciences and the American Asssociation for the Advancement of Science and a member of the National Academy of Sciences. He is a past president of the Society for Mathematical Biology.

\section{About the Medal}

The National Medal of Science is the country's highest distinction for contributions to scientific research. According to a news release from the Office of Science and Technology Policy, "The National Medal of Science honors individuals for pioneering scientific research in a range of fields, including physical, biological, mathematical, social, behavioral, and engineering sciences, that enhances our understanding of the world and leads to innovations and technologies that give the United States its global economic edge." The National Science Foundation administers the award, which was established by Congress in 1959.

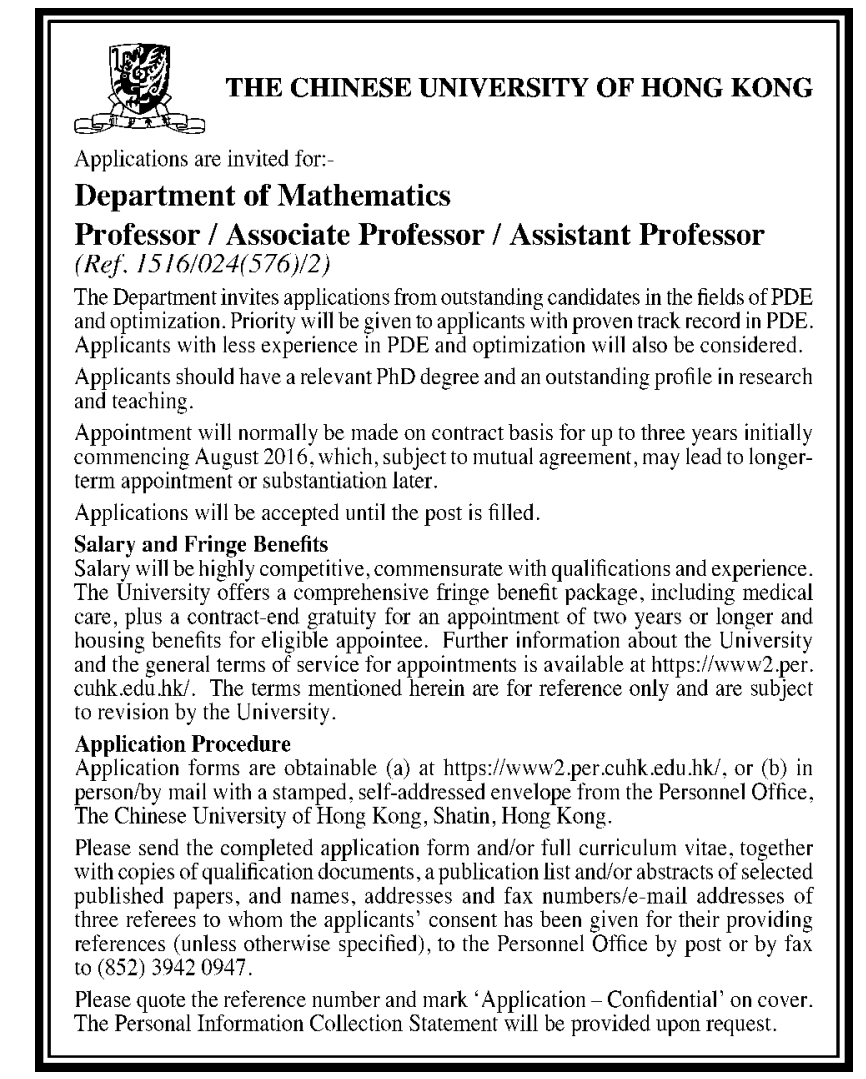

